\title{
Exenatide improves type 2 diabetes concomitant with non-alcoholic fatty liver disease
}

\author{
Exenatide melhora o diabetes tipo 2 de ocorrência concomitante \\ com doença hepática gordurosa não alcoólica
}

Hui Fan', QingRong Pan', Yuan Xu', XinChun Yang ${ }^{2}$

${ }^{1}$ Department of Endocrinology, Beijing Chaoyang Hospital, Capital University of Medical Science, Beijing, China

${ }^{2}$ Heart Center, Beijing Chaoyang Hospital, Capital University of Medical Science, Beijing, China

\author{
Correspondence to: \\ Yuan Xu \\ Department of Endocrinology, \\ Beijing Chaoyang Hospital, \\ Capital University of Medical Science, \\ Beijing 100020, China \\ xuyuan1234@126.com \\ Received on Mar/29/2013 \\ Accepted on Oct/28/2013
}

\begin{abstract}
Objective: To investigate the effects of exenatide on blood glucose, body weight and hepatic enzymes in patients with type 2 diabetes mellitus (T2DM) and concomitant non-alcoholic fatty liver disease (NAFLD). Subjects and methods: One hundred and seventeen patients with T2DM and NAFLD were randomly divided into exenatide group and metformin group. Patients were treated with exenatide and metformin, respectively, for 12 weeks. Results: After 12 weeks of treatment, body weight, body mass index (BMI), waist-to-hip ratio, HbA1c, FPG, 2-h PPG, ALT, AST, $\gamma$-GT, and hs-CRP were significantly reduced, and the AST/ALT ratio and adiponectin were markedly increased in both groups. BMI, waist-to-hip ratio, 2-h PPG, ALT, AST, $\gamma$-GT, and hs-CRP were markedly lower, and AST/ ALT ratio and adiponectin in the exenatide group were dramatically higher than in the metformin group. Conclusion: Compared with metformin, exenatide is better to control blood glucose, reduces body weight and improves hepatic enzymes, attenuating NAFLD in patients with T2DM concomitant with NAFLD. Arq Bras Endocrinol Metab. 2013;57(9):702-8
\end{abstract}

Keywords

Exenatide; metformin; non-alcoholic fatty liver disease; type 2 diabetes mellitus

\section{RESUMO}

Objetivo: Investigar os efeitos do exenatide sobre a glicose sérica, peso corporal e enzimas hepáticas em pacientes com diabetes melito tipo 2 (T2DM) e doença hepática gordurosa não alcoólica (DHGNA). Sujeitos e métodos: Um total de 117 pacientes com T2DM e DHGNA foi aleatoriamente separado em dois grupos, um tratado com exenatide e um tratado com metformina. Os pacientes foram tratados por 12 semanas. Resultados: Após 12 semanas de tratamento, o peso corporal, índice de massa corporal (IMC), relação cintura-quadril, HbA1c, FPG, glicose pós-prandial, ALT, AST, $\gamma$-GT e proteína C-reativa foram significativamente reduzidos, e a relação AST/ALT e a adiponectina aumentaram marcadamente nos dois grupos. O IMC, relação cintura-quadril, glicose pós-prandial, ALT, AST, $\gamma$-GT e proteína C-reativa foram marcadamente menores, e a relação AST/ALT e a adiponectina foram dramaticamente mais altas no grupo tratado com exenatide do que no grupo tratado com metformina. Conclusão: Comparado com a metformina, o exenatide controla melhor a glicose sérica, reduz o peso corporal e melhora as enzimas hepáticas, atenuando a DHGNA em pacientes com T2DM de ocorrência concomitante com a DHGNA. Arq Bras Endocrinol Metab. 2013;57(9):702-8

\section{Descritores}

Exenatide; metformina; doença hepática gordurosa não alcoólica; diabetes melito tipo 2

\section{INTRODUCTION}

$\mathrm{T}$ ype 2 diabetes mellitus (T2DM), obesity, and non-alcoholic fatty liver disease (NAFLD) are often identified in patients simultaneously. In patients with T2DM, the prevalence of NAFLD is as high as $75 \%$ (1). NAFLD represents the hepatic manifestation of the metabolic syndrome, which includes dyslipidemia, central obesity, hypertension, and insulin resistance (1). The prevalence of obesity and diabetes is increasing in China (2) and, thus, one may expect an increase in the incidence of NAFLD in T2DM in China. Although NAFLD and non-alcoholic steatohepatitis (NASH) are generally asymptomatic, the increase in serum alanine aminotransferase (ALT), a biochemical marker of liver injury, has been regarded as indicative of NAFLD (3). 
NAFLD can cause progressive fibrosis leading to cirrhosis and corresponding complications, including portal hypertension and liver failure (1). In addition, NAFLD is thought to be an independent determinant of cardiovascular disease (CVD), besides affecting individuals who already have multiple cardiovascular risk factors. This relationship may lead to an increased risk of undesired outcomes in this population of patients, and increased cardiovascular morbidity and mortality (4). Moreover, NAFLD is a complex syndrome that affects more than the liver.

Although the pathogenesis of NAFLD is still poorly understood, the relationship between NAFLD and insulin resistance make insulin resistance a therapeutic target. Evidence has indicated that lifestyle modifications, with weight loss and exercise, can improve, NAFLD (57 ). This has stimulated the conduction of several clinical studies in which anti-diabetic drugs, such as insulin sensitizers, including metformin, have been evaluated in NAFLD patients with insulin-resistance.

Most of findings show no clear benefit from the addition of metformin to diet or rosiglitazone, though the Alanine aminotransferase levels can be reduced by metformin treatment. It is indicated that metformin might be of benefit in the treatment of NAFLD, while associated to hypocaloric diet and weight control. The lack of benefit was because patients in the control group improved on diet, and metformin had little effect on liver histology (8). So, metformin as a hypoglycemic agent may be not reliable to alleviate NAFLD.

A new drug, glucagon-like peptide-1 analogue, such as exenatide, may be an effective anti-diabetic agent. Exenatide is a synthetic analog of exendin-4, a 39-amino-acid agonist of glucagon-like peptide 1 (GLP-1) receptor. Exendin-4 is found in the saliva of the Gila monster, Heloderma suspectum, and shares 53\% sequence homology with GLP-1 (9). GLP-1 is a gastrointestinal hormone secreted by the L cells of the intestine, which can regulate blood glucose primarily via stimulation of glucose-dependent insulin release. Therefore, exenatide may not function as a direct insulin sensitizer, but may induce clinically significant weight loss, leading to an insulin-sensitizing effect.

However, in a murine model of fatty liver, exendin-4 significantly reduced glucose levels, improved insulin sensitivity, and reduced hepatic steatosis (10). In an open-label, uncontrolled clinical trial using exenatide to assess the safety of the drug in patients with diabetes, aspartate aminotransferase (AST) and insulin sensitivity were improved over a 3.5-year follow-up period (11). Why can exenatide reverse hepatic biomarkers and type 2 diabetes with fatty liver? Is it by improving insulin sensitivity or by another way? When compared with metformin, exenatide is more effective in reducing body weight. Thus, we wonder if exenatide may serve as a drug in the treatment of NAFLD to effectively improve the hepatic biomarkers.

The present study aimed to evaluate the therapeutic effect of exenatide in NAFLD. In order to achieve this aim, plasma glucose, body weight, liver enzymology, serum $\mathrm{C}$ reactive protein (CRP), and adiponectin were evaluated after the treatment with exenatide or metfomin in T2DM patients with NAFLD.

\section{SUBJECTS AND METHODS}

\section{Patients}

From March 2009 to December 2010, a total of 134 patients with T2DM and NAFLD were enrolled in the present study. At the end of study, 117 patients were recruited for analysis, including 66 males and 51 females. The inclusion criteria were as follows: T2DM concomitant with NAFLD, poor glucose control (FBG: 6.0-10.0 mmol/L or HbAlc: 7-9\%), no acute complications or severe chronic complications of DM. The criteria for DM developed by the World Health Organization in 1999 were employed for the diagnosis of DM. Exclusion criteria were as follows: abnormal kidney function, serum $\mathrm{Cr} \geq 133 \mu \mathrm{mol} / \mathrm{L}$, presence of diseases affecting blood glucose levels (such as hyperthyroidism and hypercortisolism), treatments affecting blood glucose levels (such as steroid hormones), pregnancy, breast-feeding, and patients with history of chronic drinking. All patients were diagnosed with T2DM concomitant with NAFLD; $52 \%$ of patients had abnormal liver function (ALT > upper limit of normal; ALT > 2.5 times of upper limit of normal in 46 patients). Ultrasonography was carried out for the diagnosis of fatty liver (Philips iu 22; C5-2 probe) by two medical sonographers, based on the following criteria: 1) enlarged liver, increase and intensification in echoes and bright spots (bright liver); 2) unclear or absent intrahepatic blood vessels; 3 ) reduced or attenuated deep echoes. Patients who met the diagnostic criteria and had no history of drinking, acute fatty liver, drug-induced fatty liver, hepatitis $\mathrm{B}$, and hepatitis $\mathrm{C}$ were recruited for the present study. 


\section{Therapeutic regimen}

Patients were divided into two age- and gender-matched groups. All the patients underwent lifestyle interventions. In Group A, patients $(n=49)$ were treated with exenatide, and in Group B ( $\mathrm{n}=68)$, with metformin. The therapeutic regimen was as follows: Group A: Exenatide injection (Eli Lilly and Company, USA) was administrated from week 1 to week 4 at $5 \mu \mathrm{g}$ (bid), and from week 5 to week 12 at $10 \mu$ g (bid). Group B: metformin was initially administered at $0.5 \mathrm{~g}$ (bid). The dose of metformin (Sino-American Shanghai Squibb Pharmaceutical Ltd.) was adjusted to a maximum of $2.0 \mathrm{~g} / \mathrm{d}$ on the basis of FPG and 2-h PPG.

\section{Observations}

The measurement of height, body weight, waist-to-hip ratio and blood pressure, ultrasonography of the liver, gallbladder and spleen, and detection of $\mathrm{HbAlc}, \mathrm{FPG}$, 2-h PPG, FIN, TG, HDL-C, LDL-C, Tch-C, ALT, AST, $\gamma$-GT, hs-CRP and adiponectin were done at baseline and after 12 weeks of treatment. The hs-CRP was detected with Latex-enhanced immune turbidimetric method (Orion, Fineland), and serum adiponectin with radioimmunoassay (Linco, USA). Patients were re-examined after 4 weeks of the treatment, when FBG and 2-h PPG at each meal were measured.

\section{Statistical analysis}

Quantitative data were expressed as mean \pm standard deviation $(\bar{\chi} \pm S D)$. Statistical analysis was done with SPSS version 17.0. Means before and after treatment were compared between the two groups with independent $\mathrm{t}$ test or rank sum test. Means before and after treatment in each group were compared with paired $t$ test or rank sum test. Qualitative data were compared with chi-square test. Two-sided $\mathrm{P}$ value of $<0.05$ was considered statistically significant. HOMA-IR was calculated as follow: fasting blood glucose vs. fasting blood insulin/22.5.

\section{RESULTS}

\section{Characteristics of patients at baseline}

A total of 144 patients were recruited for the present study, and 27 of them withdrew from the study or were lost to follow-up. In the end, 117 patients completed this study. Mean age was $52.35 \pm 11.83$ years, mean body weight was $78.59 \pm 11.03 \mathrm{~kg}$, and mean body mass index (BMI) was $27.02 \pm 4.43 \mathrm{~kg} / \mathrm{m}^{2}$. These patients were characterized by mild to moderate increase in ALT, AST and $\gamma$-GT, as well as reduction in AST/ ALT.

At baseline, there were no marked differences in age, body weight, WHR, HbAlc, FPG, 2-h PPG, TG, HDL-C, LDL-C, Tch-C, ALT, AST, $\gamma$-GT, adiponectin, and hs-CRP (Table 1 ).

Table 1. Demographics of patients with T2DM and NAFLD at baseline

\begin{tabular}{|c|c|c|c|c|}
\hline & $\begin{array}{l}\text { Group A } \\
(n=49)\end{array}$ & $\begin{array}{l}\text { Group B } \\
(n=68)\end{array}$ & $\mathbf{t}$ & $\mathbf{P}$ \\
\hline Age (yr) & $51.02 \pm 10.10$ & $54.68 \pm 12.14$ & -1.68 & 0.10 \\
\hline Gender (M/F) & $28 / 21$ & $38 / 30$ & 0.018 & 0.89 \\
\hline Body weight (kg) & $80.61 \pm 11.23$ & $78.58 \pm 10.76$ & 2.122 & 0.168 \\
\hline BMI & $28.18 \pm 1.86$ & $27.61 \pm 1.77$ & 1.58 & 0.12 \\
\hline WHR & $0.98 \pm 0.08$ & $0.97 \pm 0.06$ & -0.06 & 0.95 \\
\hline ALT (U/L) & $65.74 \pm 18.13$ & $65.81 \pm 17.58$ & -0.02 & 0.99 \\
\hline AST (U/L) & $35.92 \pm 12.27$ & $34.28 \pm 13.69$ & 0.62 & 0.54 \\
\hline AST/ALT & $0.60 \pm 0.06$ & $0.60 \pm 0.06$ & 0.05 & 0.96 \\
\hline$\gamma-\mathrm{GT}(\mathrm{U} / \mathrm{L})$ & $70.21 \pm 21.54$ & $62.65 \pm 19.02$ & 1.79 & 0.08 \\
\hline HbA1c (\%) & $8.14 \pm 0.51$ & $8.09 \pm 0.59$ & -0.86 & 0.59 \\
\hline $\mathrm{FPG}(\mathrm{mmol} / \mathrm{L})$ & $8.45 \pm 0.64$ & $8.41 \pm 0.69$ & 0.34 & 0.73 \\
\hline 2-h PG (mmol/L) & $15.09 \pm 5.42$ & $14.64 \pm 5.12$ & 0.81 & 0.42 \\
\hline $\mathrm{TG}(\mathrm{mmol} / \mathrm{L})$ & $2.47 \pm 1.49$ & $2.51 \pm 1.73$ & -1.01 & 0.32 \\
\hline TCH-C (mmol/L) & $5.03 \pm 0.97$ & $5.15 \pm 0.95$ & -0.62 & 0.54 \\
\hline LDL-C (mmol/L) & $2.56 \pm 0.83$ & $2.62 \pm 0.82$ & -1.57 & 0.12 \\
\hline $\mathrm{HDL}-\mathrm{C}(\mathrm{mmol} / \mathrm{L})$ & $1.05 \pm 0.24$ & $0.99 \pm 0.20$ & 1.34 & 0.18 \\
\hline hs-CRP (mg/L) & $3.14 \pm 0.58$ & $3.16 \pm 0.68$ & -0.18 & 0.86 \\
\hline Adiponectin (mg/L) & $8.43 \pm 1.01$ & $8.13 \pm 1.23$ & 1.27 & 0.21 \\
\hline HOMA-IR & $3.05 \pm 0.55$ & $2.94 \pm 0.59$ & 0.95 & 0.34 \\
\hline
\end{tabular}

\section{Variables after the 12-week treatment}

In the treatment, the major side effects included nausea, upper abdominal discomfort and decreased appetite. Three patients treated with metformin developed diarrhea. Patients were tolerant to these side effects within 2-3 weeks. At 12 weeks of treatment, body weight, BMI, WHR, HbAlc, FPG, 2-h PPG, ALT, AST and $\gamma$-GT reduced significantly when compared with the values observed before treatment. There were no marked differences in HbAlc, FPG, HOMA-IR, TG, HDL-C, LDL-C, and Tch-C between two groups after treatment. However, body weight $(76.09 \pm 9.85$ vs. $77.22 \pm 10.15, \mathrm{P}=0.02)$, BMI $(25.87 \pm 1.48$ vs. 26.91 $\pm 1.79, \mathrm{P}=0.02), \mathrm{WHR}(0.95 \pm 0.05$ vs. $0.96 \pm 0.04$, $\mathrm{P}=0.01), \operatorname{ALT}(39.82 \pm 14.05$ vs. $51.48 \pm 18.89, \mathrm{P}=$ 
$0.00), \operatorname{AST}(25.61 \pm 7.87$ vs. $31.54 \pm 10.75, \mathrm{P}=0.01)$, AST/ALT $(0.81 \pm 0.17$ vs. $0.69 \pm 0.15, \mathrm{P}=0.00)$, $\gamma$-GT $(47.53 \pm 15.80$ vs. $53.44 \pm 15.00, \mathrm{P}=0.05)$, hs-CRP $(2.18 \pm 0.34$ vs. $2.69 \pm 0.53, \mathrm{P}=0.01)$, and $2-\mathrm{h}$ PPG $(10.31 \pm 3.17$ vs. $12.05 \pm 4.03, \mathrm{P}=0.036)$ were markedly reduced, and adiponectin increased dramatically in the exenatide group when compared with the metformin group (Table 2).

Table 2. Variables in two groups after 12 weeks of treatment

\begin{tabular}{|c|c|c|c|c|}
\hline Variables & $\begin{array}{c}\text { Exenatide } \\
\text { group }(n=49)\end{array}$ & $\begin{array}{c}\text { Metformin } \\
\text { group }(n=68)\end{array}$ & $\mathbf{t}$ & $\mathbf{P}$ \\
\hline Body weight (kg) & $76.09 \pm 9.85$ & $77.22 \pm 10.15$ & -2.764 & $0.02^{*}$ \\
\hline BMl & $25.87 \pm 1.48$ & $26.91 \pm 1.79$ & -2.28 & $0.02^{*}$ \\
\hline WHR & $0.95 \pm 0.05$ & $0.96 \pm 0.04$ & -2.59 & $0.009^{*}$ \\
\hline ALT (U/L) & $39.82 \pm 14.05$ & $51.48 \pm 18.89$ & -3.36 & $0.000^{* *}$ \\
\hline AST (U/L) & $25.61 \pm 7.87$ & $31.54 \pm 10.75$ & 2.62 & $0.012^{*}$ \\
\hline AST/ALT & $0.81 \pm 0.17$ & $0.69 \pm 0.15$ & 4.34 & $0.00^{*}$ \\
\hline$\gamma$-GT (U/L) & $47.53 \pm 15.80$ & $53.44 \pm 15.00$ & -1.92 & $0.047^{\star}$ \\
\hline HbA1c (\%) & $7.15 \pm 0.36$ & $7.16 \pm 0.58$ & -1.73 & 0.082 \\
\hline $\mathrm{FPG}(\mathrm{mmol} / \mathrm{L})$ & $7.11 \pm 0.68$ & $7.32 \pm 0.76$ & -1.52 & 0.131 \\
\hline 2-h PG (mmol/L) & $10.31 \pm 3.17$ & $12.05 \pm 4.03$ & -2.43 & $0.024^{*}$ \\
\hline $\mathrm{TG}(\mathrm{mmol} / \mathrm{L})$ & $1.84 \pm 0.51$ & $1.78 \pm 0.60$ & 0.95 & 0.345 \\
\hline TCH-C (mmol/L) & $4.88 \pm 1.51$ & $4.82 \pm 1.50$ & -0.09 & 0.934 \\
\hline LDL-C (mmol/L) & $2.42 \pm 0.52$ & $2.45 \pm 0.55$ & -0.029 & 0.687 \\
\hline $\mathrm{HDL}-\mathrm{C}(\mathrm{mmol} / \mathrm{L})$ & $1.06 \pm 0.22$ & $1.03 \pm 0.23$ & 1.08 & 0.084 \\
\hline hs-CRP (mg/L) & $2.18 \pm 0.34$ & $2.69 \pm 0.53$ & -3.21 & $0.023^{*}$ \\
\hline Adiponectin (mg/L) & $10.44 \pm 3.29$ & $8.48 \pm 2.67$ & 3.45 & $0.000^{\star \star}$ \\
\hline HOMA-IR & $2.47 \pm 0.44$ & $2.48 \pm 0.51$ & -0.10 & 0.923 \\
\hline
\end{tabular}

${ }^{*} \mathrm{P} \leq 0.05 ;{ }^{* *} \mathrm{P} \leq 0.01$.

\section{Body weight and biochemical variables before and} after treatment

In both groups, body weight, BMI, WHR, HbAlc, FPG, 2-h PPG, HOMA-IR, ALT, AST, $\gamma$-GT, TG, and hs-CRP reduced significantly; HDL-C, LDL-C, and Tch-C remained unchanged; and AST/ALT and adiponectin increased dramatically when compared with values observed before treatment. In the exenatide group, reductions in body weight $(4.16 \pm 5.32 \mathrm{vs} .1 .98$ $\pm 3.28), \mathrm{BMI}(1.3 \mathrm{l} \pm 0.98$ vs. $0.69 \pm 0.94), \mathrm{WHR}(0.04$ \pm 0.02 vs. $0.02 \pm 0.02), \operatorname{ALT}(27.32 \pm 15.96 v s .12 .85 \pm$ $11.38), \operatorname{AST}(7.89 \pm 7.8$ vs. $75.11 \pm 6.98), \gamma-$ GT $(26.48$ \pm 17.34 vs. $10.26 \pm 14.11)$, and hs-CRP $(0.89 \pm 0.59$ vs. $0.61 \pm 0.54)$, and increases in AST/ALT $(0.23 \pm$ 0.08 vs. $0.11 \pm 0.09)$, adiponectin $(1.86 \pm 2.22$ vs. 0.76 $\pm 1.30)$ and $2-\mathrm{h} \mathrm{PPG}(4.16 \pm 3.01$ vs. $2.63 \pm 2.28)$ were more obvious than those in the metformin group. However, reductions in FPG, HbAlc and HOMA-IR were comparable between two groups (Table 3 ).

Table 3. Variables after 12 weeks of treatment in the two groups

\begin{tabular}{|c|c|c|c|c|}
\hline \multirow{2}{*}{ Factors } & \multicolumn{4}{|c|}{ Changes in variables } \\
\hline & $\begin{array}{c}\text { Exenatide } \\
\text { group }(n=49)\end{array}$ & $\begin{array}{c}\text { Metformin } \\
\text { group }(n=68)\end{array}$ & $\mathbf{t}$ & $\mathbf{P}$ \\
\hline Body weight (kg) & $4.16 \pm 5.32$ & $1.98 \pm 3.28$ & 2.01 & $0.000^{* *}$ \\
\hline BMI & $1.31 \pm 0.98$ & $0.69 \pm 0.94$ & 3.22 & $0.000^{* \star}$ \\
\hline WHR & $0.04 \pm 0.02$ & $0.02 \pm 0.02$ & 3.08 & $0.000^{\star \star}$ \\
\hline ALT (U/L) & $27.32 \pm 15.96$ & $12.85 \pm 11.38$ & 5.73 & $0.002^{\star \star}$ \\
\hline AST (U/L) & $7.89 \pm 7.87$ & $5.11 \pm 6.98$ & 2.03 & $0.048^{*}$ \\
\hline AST/ALT & $-0.23 \pm 0.08$ & $-0.11 \pm 0.09$ & -1.02 & $0.043^{*}$ \\
\hline$\gamma-\mathrm{GT}(\mathrm{U} / \mathrm{L})$ & $26.48 \pm 17.34$ & $10.26 \pm 14.11$ & 5.98 & $0.000^{\star \star}$ \\
\hline HbA1c (\%) & $0.91 \pm 0.65$ & $0.89 \pm 0.57$ & 1.30 & 0.203 \\
\hline $\mathrm{FPG}(\mathrm{mmol} / \mathrm{L})$ & $1.09 \pm 0.83$ & $1.12 \pm 0.80$ & -0.08 & 0.581 \\
\hline $2 \mathrm{hPG}(\mathrm{mmol} / \mathrm{L})$ & $4.16 \pm 3.01$ & $2.63 \pm 2.28$ & 2.03 & $0.022^{*}$ \\
\hline $\mathrm{TG}(\mathrm{mmol} / \mathrm{L})$ & $0.31 \pm 1.44$ & $0.33 \pm 1.06$ & -0.11 & 0.904 \\
\hline TCH-C (mmol/L) & $0.29 \pm 0.71$ & $0.26 \pm 0.80$ & 0.17 & 0.939 \\
\hline LDL-C (mmol/L) & $0.19 \pm 0.63$ & $0.16 \pm 0.71$ & 0.20 & 0.646 \\
\hline HDL-C (mmol/L) & $0.02 \pm 0.50$ & $0.02 \pm 0.61$ & 0.10 & 0.827 \\
\hline hs-CRP (mg/L) & $0.89 \pm 0.59$ & $0.61 \pm 0.54$ & 2.59 & $0.018^{* \star}$ \\
\hline Adiponectin (mg/L) & $-1.86 \pm 2.22$ & $-0.76 \pm 1.30$ & -7.37 & $0.001^{* \star}$ \\
\hline HOMA-IR & $0.57 \pm 0.36$ & $0.56 \pm 0.49$ & 0.89 & 0.367 \\
\hline
\end{tabular}

${ }^{*} P \leq 0.05 ;{ }^{* *} P \leq 0.01$.

\section{HbA1c and ALT after treatment}

After the 12-week treatment, the proportion of patients with $\mathrm{HbAlc}<7 \%$ was comparable between two groups, but the proportion of patients with normal ALT level $(<40 \mathrm{U} / \mathrm{L})$ in the exenatide group was significantly lower than in the metformin group. Re-examination revealed that the percentage of patients with concomitant NAFLD in the exenatide group was slightly lower than in the metformin group, which might be related to the short-term treatment or small sample size (Table 4).

\section{DISCUSSION}

With the elevation in the standard of living, prevalence of obesity and T2DM increases. Studies have shown that obesity may increase the risk of DM, as well as cardiovascular diseases and NAFLD. In clinical practice, we usually encounter patients that develop T2DM, obesity and NAFLD simultaneously $(12,13)$. Insulin resistance may compromise the ability of insulin to 
Table 4. Proportion of patients with $\mathrm{HbA} 1 \mathrm{C}>7 \%$ and $\mathrm{ALT}<40 \mathrm{U} / \mathrm{L}$

\begin{tabular}{lcccccc}
\hline & HbA1c $<$ 7\% & HbA1c > 7\% & $\begin{array}{c}\text { Concomitant } \\
\text { NAFLD }\end{array}$ & Absence of NAFLD & ALT < 40 U/L & ALT > 40 U/L \\
\hline Exenatide $(n=49)$ & $18(36.7 \%)$ & $31(63.3 \%)$ & 39 & 10 & $27(55.1 \%)$ & $22(44.9 \%)$ \\
Metformin $(n=68)$ & $28(41.2 \%)^{*}$ & $40(58.8 \%)^{*}$ & 61 & 7 & $18(26.5 \%)$ & $50(73.5 \%)$ \\
Value & & 0.24 & & 2.35 & & 9.86 \\
$\mathrm{P}$ & & 0.62 & & 0.13 & & $0.02^{*}$ \\
\hline
\end{tabular}

${ }^{*} \mathrm{P}<0.05$ between two groups after treatment.

inhibit lipase, which leads to the increased degradation of peripheral adipose tissue and elevated free fatty acid. Then, oxidation and consumption of free fatty acid are insufficient, resulting in lipoylation of fatty acid into triglycerides, and increased triglyceride levels. Triglyceride is then used to synthesize fat, which deposits in the liver, resulting in fatty liver. The deposition of fat in the liver may reduce the clearance of insulin in the liver, which aggravates the peripheral and hepatic insulin resistance, resulting in a positive feedback amplification. Thus, hepatic insulin resistance has been regarded as an important characteristic of NAFLD. In addition, the imbalanced activities of adipocytokines might be a cause of NAFLD progression after body weight loss and reduction in body fat.

The adipose tissue is not only an important organ for energy storage, but also a key endocrine organ. It can produce some adipocytokines via autocrine and paracrine mechamisms. Of these adipocytokines, leptin, adiponectin, resistin, tumor necrosis factor, and interleukin have been found to be closely related to NAFLD. In addition, hs-CRP is another important inflammatory factor and has been regarded as a pivotal factor that can predict chronic inflammatory reaction, metabolic syndrome, and cardiovascular diseases (14). Adiponectin is one of proteins with high expression in the adipose tissues, which can inhibit inflammation. Arvaniti and cols. (15) found that hypoadiponectinemia could induce fat deposition in the liver and the progression of fatty hepatitis, and it is the most important adipocytokine in the initiation and progression of NAFLD. Thus, insulin resistance, increase in inflammatory cytokines, such as hs-CRP, and reduction in adiponectin act synergistically to promote the occurrence and development of T2DM and NAFLD.

The majority of patients with NAFLD have no evident clinical symptoms and only present increase in ALT and/or AST. The mild to moderate increase in ALT and $\gamma$-GT and reduction in AST/ALT have been considered as characteristics closely related to NAFLD,
T2DM, and metabolic syndrome (16). Currently, some studies reveal that NAFLD is closely related to the increased risk of cardiovascular disease (17). In NAFLD patients, severe insulin resistance, sustained hyperinsulinism, disordered lipid metabolism (increase in $\mathrm{TG}$, reduction in HDL-C, and elevation in LDL-C) and subclinical inflammatory state may serve as risk factors to promote the occurrence and development of atherosclerosis $(17,18)$. On the other hand, NAFLD may result in progressive hepatitis, hepatic fibrosis or cirrhosis, or even liver failure (19). Thus, for T2DM patients with concomitant NAFLD, therapy targeting insulin resistance may improve NAFLD. For example, body weight loss may not only reduce blood glucose, but may also be beneficial for blood pressure, blood lipids and the liver, leading to reduction in risk of cardiovascular diseases.

In previous therapies for T2DM (such as application with insulin and insulin secretagogues), hypoglycemia and body weight increase were usually encountered in clinical practice. In a large prospective study (ACCORD study), the investigators have proposed caution on the goal and safety of lowering glucose levels (20). In addition, previous therapies often fail to improve insulin resistance and have limitations in improving cardiovascular complications. Besides lifestyle interventions, such as exercise and diet control, metformin and thiazolidinedione have been applied in the treatment of DM, but their therapeutic efficacy is not ideal. A recent meta-analysis reveals that lifestyle intervention is comparable to metformin in the improvement of liver histology and hepatic enzymes in NAFLD patients (21). Thus, we pose the question if GLP-1 may be a promising anti-DM drug.

Exenatide is an incretin analogue and can mimic the actions of GLP-1. For example, it can promote the glucose-dependent insulin secretion, inhibit the inappropriate secretion of glicentin, delay gastric emptying, and suppress appetite to reduce blood glucose and body weight $(22)$. In a randomized, controlled study 
on T2DM, exenatide was superior to insulin glargine in improving the risk factors of cardiovascular diseases (such as adiponectin and hs-CRP) (23). It is a question to be answered whether exenatide is superior to metformin in improving NAFLD and related biochemical variables.

In the present study, T2DM patients with NAFLD were recruited, and the effects of exenatide and metformin on the blood glucose, NAFLD-related variables (such as ALT, AST, and $\gamma$-GT) were compared. In addition, ultrasonography was done to evaluate the fatty liver before and after treatment. Our results demonstrated that exenatide was comparable to metformin in the reduction of blood glucose, but it was superior to metformin in reducing body weight, decreasing WHR, and improving hepatic enzymes. Although ultrasonography showed that the proportion of patients with improvement of fatty liver was comparable between two groups, possibly due to short term treatment and/or small sample size, exenatide had a slightly better ability to improve the fatty liver. HOMA-IR was employed to evaluate insulin resistance, and results did not indicate differences between the two groups. Thus, exenatide has special effect on the liver, which is different from metformin. Moreover, exenatide is better to control 2-h PPG when compared with metformin, and our results showed that serum adiponectin in patients treated with exenatide was higher than that in metformin-treated patients, but hs-CRP was reduced in exenatide-treated patients when compared with metformin-treated patients. Whether exenatide has a sustained effect in improving abdominal obesity or whether exenatide interrupts the vicious circle between insulin resistance and NAFLD is still unclear. The failure of metformin to improve NAFLD might be related to the absence of improvement of adiponectin and hs-CRP, which suggests that metformin has no ability to improve oxidative stress induced by fatty acid deposition and inflammation in the liver (24). The GLP-1 analogue may improve fatty liver and insulin resistance. This may be explained by the fact that GLP-1 may directly act on hepatocytes and activate the expression of genes related to the $\beta$-oxidation of fatty acids and insulin sensitivity (such as PPAR- $\gamma$ and PPAR- $\alpha$ ), which lead to the improvement of insulin resistance in the NAFLD patients (25).

Currently, exenatide treatment is costly, which significantly limits its wide application in clinical practice. However, most of T2DM patients with abdominal obesity develop NAFLD characterized by evident increase in ALT, which influences liver function, increases the risk for hepatic cirrhosis and liver failure, and limits the application of oral anti-diabetic drugs. In addition, application of insulin may increase body weight. Thus, short term application of exenatide may interrupt this vicious cycle to control blood glucose, reduce body weight, and improve the liver function.

Therefore, for T2DM patients with NAFLD, exenatide as a GLP-1 receptor agonist may not only reduce blood glucose and body weight, but also improve liver enzymes, attenuating NAFLD. For NAFLD, the improvement of liver histology is a gold standard in the evaluation of therapeutic efficacy. However, liver histology was not conducted in the present study. In future studies, long-term treatment with exenatide and a large sample size are required to confirm the effects of exenatide on NAFLD, insulin resistance, and atherosclerosis and to determine whether exenatide can be of benefit to NAFLD patients.

Disclosure: no potential conflict of interest relevant to this article was reported.

\section{REFERENCES}

1. Sass DA, Chang P, Chopra KB. Nonalcoholic fatty liver disease: a clinical review. Dig Dis Sci. 2005;50 (1):171-80.

2. Yang W, Lu J, Weng J, Jia W, Ji L, Xiao J, et al. Prevalence of diabetes among men and women in China. $\mathrm{N}$ Engl $\mathrm{J}$ Med. 2010;362(12):1090-101.

3. Leavens KF, Birnbaum MJ. Insulin signaling to hepatic lipid metabolism in health and disease. Crit Rev Biochem Mol Biol. 2011;46:200-15.

4. Targher G, Day CP, Bonora E. Risk of cardiovascular disease in patients with nonalcoholic fatty liver disease. $\mathrm{N}$ Engl J Med. 2010;363:1341-50.

5. Huang MA, Greenson JK, Chao C, Anderson L, Peterman D, Jacobson $J$, et al. One-year intense nutritional counseling results in histological improvement in patients with nonalcoholic steatohepatitis: a pilot study. Am J Gastroenterol. 2005;100(5):1072-81.

6. Wang RT, Koretz RL, Yee HF Jr. Is weight reduction an effective therapy for nonalcoholic fatty liver? A systematic review. Am J Med. 2003;115(7):554-9.

7. Kadayifci A, Merriman RB, Bass NM. Medical treatment of nonalcoholic steatohepatitis. Clin Liver Dis. 2007;11(1):119-40.

8. Musso G, Gambino R, Cassader M. A meta-analysis of randomized trials for the treatment of nonalcoholic fatty liver disease. Hepatology. 2010;52(1):79-104.

9. Eng J, Kleinman WA, Singh L, Singh G, Raufman JP. Isolation and characterization of exendin-4, an exendin-3 analogue, from Heloderma suspectum venom. Further evidence for an exendin receptor on dispersed acini from guinea pig pancreas. J Biol Chem. 1992;267(11):7402-5.

10. Ding X, Saxena NK, Lin S, Gupta NA, Anania FA. Exendin-4, a glucagon-like protein-1 (GLP-1) receptor agonist, reverses hepatic steatosis in ob/ob mice. Hepatology. 2006;43(1):173-81. 
11. Klonoff DC, Buse JB, Nielsen LL, Guan X, Bowlus CL, Holcombe $\mathrm{JH}$, et al. Exenatide effects on diabetes, obesity, cardiovascular risk factors and hepatic biomarkers in patients with type 2 diabetes treated for at least 3 years. Curr Med Res Opin. 2008;24(1):275-86.

12. Bray GA. Medical consequences of obesity. J Clin Endocrinol Metab. 2004;89(6):2583-9.

13. Mokdad AH, Ford ES, Bowman BA, Dietz WH, Vinicor F, Bales VS, et al. Prevalence of obesity, diabetes, and obesity-related health risk factors, 2001. JAMA. 2003;289(1):76-9.

14. Zimmermann E, Anty R, Tordjman J, Verrijken A, Gual P, Tran A, et al. $C$-reactive protein levels in relation to various features of nonalcoholic fatty liver disease among obese patients. J Hepatol. 2011;55(3):660-5.

15. Arvaniti VA, Thomopoulos KC, Tsamandas A, Makri M, Psyrogiannis A, Vafiadis $G$, et al. Serum adiponectin levels in different types of non alcoholic liver disease. Correlation with steatosis, necroinflammation and fibrosis. Acta Gastroenterol Belg. 2008;71(4):355-60.

16. Schindhelm RK, Diamant M, Dekker JM, Tushuizen ME, Teerlink T, Heine RJ. Alanine aminotransferase as a marker of non-alcoholic fatty liver disease in relation to type 2 diabetes mellitus and cardiovascular disease. Diabetes Metab Res Rev. 2006;22(6):437-43.

17. Hanley AJ, Williams K, Festa A, Wagenknecht LE, D'Agostino RB $\mathrm{Jr}$, Haffner SM. Liver markers and development of the metabolic syndrome: the insulin resistance atherosclerosis study. Diabetes. 2005;54(11):3140-7.
18. Pacana T, Fuchs M. The cardiovascular link to nonalcoholic fatty liver disease: a critical analysis. Clin Liver Dis. 2012;16(3):599-613.

19. Ali R, Cusi K. New diagnostic and treatment approaches in nonalcoholic fatty liver disease (NAFLD). Ann Med. 2009;41(4):265-78.

20. American Gastroenterological Association. American Gastroenterological Association medical position statement: nonalcoholic fatty liver disease. Gastroenterology. 2002;123(5):1702-4.

21. Buse JB, Ginsberg HN, Bakris GL, Clark NG, Costa F, Eckel R, et al.; American Heart Association; American Diabetes Association. Primary prevention of cardiovascular diseases in people with diabetes mellitus: a scientific statement from the American Heart Association and the American Diabetes Association. Diabetes Care. 2007;30:162-72.

22. Gallwitz B. Glucagon-like peptide-1 analogues for Type 2 diabetes mellitus: current and emerging agents. Drugs. 2011;71(13):1675-88.

23. Bunck MC, Diamant $M$, Eliasson $B$, Cornér $A$, Shaginian RM, Heine RJ, et al. Exenatide affects circulating cardiovascular risk biomarkers independently of changes in body composition. Diabetes Care. 2010;33(8):1734-7.

24. Van Wagner LB, Rinella ME. The role of insulin-sensitizing agents in the treatment of nonalcoholic steatohepatitis. Therap Adv Gastroenterol. 2011;4(4):249-63.

25. Svegliati-Baroni G, Saccomanno S, Rychlicki C, Agostinelli L, De Minicis S, Candelaresi C, et al. Glucagon-like peptide-1 receptor activation stimulates hepatic lipid oxidation and restores hepatic signalling alteration induced by a high-fat diet in nonalcoholic steatohepatitis. Liver Int. 2011;31(9):1285-97. 